\title{
Effects on recall of signals to text organization
}

\author{
ROBERT F. LORCH, JR. \\ University of Kentucky, Lexington, Kentucky
}

(Susan M. Belmore, Sponsor)

\begin{abstract}
College subjects read and recalled one of two versions of the same text. One version of the text contained signals to both the general organization of the text and to the organization of specific sentences in the text; the alternate version did not contain organizational signals. Overall recall was better for the text with signals than for the text without signals because recall of specifically signaled information was aided by signaling. The magnitude of the signaling effect was related to subjects' text recall abilities: Signaling effects were larger for better recallers than for poorer recallers.
\end{abstract}

In order to comprehend a text, a reader must represent the structure of the text as well as its content (Kintsch \& van Dijk, 1978). In processing a text's structure, a reader must recognize both the relative importance of text propositions and the organization of those propositions. A writer can facilitate the reader's task by using a variety of signals to indicate a text's structure and to emphasize particular information (Meyer, 1975; van Dijk, 1979).

A text signal is a writing device that does not add new content to a text; rather it emphasizes certain aspects of a text (Meyer, 1975). There are many different kinds of signaling devices. Some signals emphasize specific text content (e.g., "It is important to stress. .."), whereas other signals emphasize the organization of information in a text (e.g., numbering). Signals may operate at high levels (e.g., titles) or low levels (e.g., underlining) of text structure. Finally, signals may involve typographical cues which distinguish them from the text per se (e.g., headings), or they may be embedded in the text (e.g., preview sentences). The present investigation is concerned with the combined effects of two text-embedded signaling devices which function to emphasize text organization. The issue of primary concern is how the organizational signals affect text recall. The results of the experiment also allow consideration of the nature of individual differences in the effects of organizational signals.

It is well known that organization is an important determinant of recall of word lists (e.g., Tulving \& Pearlstone, 1966) and of text (e.g., Frase, 1969). Thus, signaling the organization of a text should facilitate its subsequent recall by making that organization more explicit and salient to the reader. One way to emphasize text

This research was supported by a University of Kentucky Research Foundation Grant and by a UKRF Faculty Summer Fellowship. I would like to thank David A. Balota, Susan M. Belmore, Melissa J. Himelein, and Elizabeth Pugzles Lorch for their careful critiques of earlier versions of this paper. Requests for reprints should be addressed to: R. F. Lorch, Jr., Department of Psychology, Kastle Hall, University of Kentucky, Lexington, KY 40506-0044. organization is with signals that are physically distinct from the text, such as headings (e.g., "Results"). There is evidence that headings selectively aid recall of important text information (Brooks, Dansereau, Spurlin, \& Holley, 1983; Loman \& Mayer, 1983). Another way to signal organization is with preview sentences, logical connectives, and other devices embedded in the text. In contrast to findings on the effects of headings, studies of text-embedded organizational signals have found little evidence of effectiveness. Several studies have failed to demonstrate that signaling text structure facilitates overall recall (Britton, Glynn, Meyer, \& Penland, 1982; Irwin, 1982; Meyer, 1975). Those studies reporting effects found better recall only for signaled information and only for underachieving or poor readers (Marshall \& Glock, 1978; Meyer, Brandt, \& Bluth, 1980).

Previous failures to demonstrate effects of textembedded organizational signals may be due to the use of overall recall as the dependent measure. When other types of text signals have been studied, the presence of signals has often been found to facilitate recall of signaled information while inhibiting recall of unsignaled information (Fowler \& Barker, 1974; Glynn \& DiVesta, 1979; Loman \& Mayer, 1983). The current experiment thus examined the effects of text-embedded organizational signals on signaled and unsignaled information. Subjects read and free recalled a text that either did or did not include signals. The signaled version of the text included both preview sentences indicating the major sections of the text (e.g., "Let us consider the first piece of evidence."), and numbering signals indicating the organization of specific facts presented in the text (e.g., “(1) First, ... .). Subjects' overall recalls were broken down into two components. First, recall was scored for content identified as being specifically indicated by numbering signals (specific recall). Second, recall was assessed for the remaining text content (nonspecific recall). If the organizational signals affect recall selectively, then specific recall scores should be higher for the signaled text, and nonspecific recall scores should be higher for the unsignaled text. 
A secondary concern of the current study is whether readers of different text-recall abilities respond differently to text signals. Two competing hypotheses may be proposed to explain how signaling effects might vary with differences in text-recall ability. One possibility is that good recallers do not need the help of text signals to process a text's organization, whereas poor recallers are deficient in organizational skills and, thus, benefit from devices that make a text's organization more salient (Meyer et al., 1980). This hypothesis predicts that signals will aid poor recallers more than good recallers. The second hypothesis is that differences in sensitivity to organizational cues are an important component of differences in text-recall ability (Brown, 1980). Good recallers are good in part because they attend to organizational signals as they read. This hypothesis predicts that good recallers will benefit more from signals than poor recallers. In order to examine individual differences in signaling effects, a measure of text recall performance will be correlated with a measure of the effects of signaling. If a relationship is demonstrated, the nature of the relation will be observed in order to determine whether signals are more beneficial to good or poor text recallers.

\section{METHOD}

\section{Subjects}

Subjects were 113 introductory psychology students at the University of Kentucky who received credit for participation in the experiment.

\section{Materials}

The stimulus text was a story about the kidnapping of Charles Lindbergh's baby (Wallechinsky \& Wallace, 1978). In addition, the NelsonDenny (Brown, Nelson, \& Denny, 1973) vocabulary test (100-item, multiple choice) was used in a distractor task.

Two versions of the text were written. Each version was typed single spaced on two pages. The signaled version contained preview sentences at the start of most paragraphs. These sentences clearly indicated the topic of the subsequent section of the text without contributing new information to their respective paragraphs. In addition, there were two places in the text that involved a listing of evidence relevant to the kidnapping case. The organization of this evidence was signaled by the use of an introductory sentence and by numbering of each successive piece of evidence. The unsignaled version of the text differed from the signaled version in that the preview sentences were deleted. Also, the numbering signals were deleted and the initial parts of the corresponding sentences were rewritten where necessary to maintain grammaticality.

\section{Procedure}

Subjects were run in groups of between 5 and 40 subjects in sessions lasting approximately $45 \mathrm{~min}$. Subjects were assigned at random to the two experimental conditions ( 56 in the signal condition; 57 in the no signal condition). They were instructed to read the text once at their own pace in preparation for a subsequent recall test. When all subjects had read the story, the texts were collected and the vocabulary test was administered for $10 \mathrm{~min}$. Finally, subjects gave a written free recall of the story. The recall was self paced.

\footnotetext{
Scoring

The unsignaled version of the text was analyzed into 43 idea units, one idea unit for each major sentence clause in the text. Each subject's recall protocol was scored with respect to the number of idea units correctly reproduced from the text. Overall recall was broken down into: (1) recall of the 9 idea units that were specifically marked by numbering in the text (specific recall score); and (2) recall of the remaining
}

34 idea units (nonspecific recall score). The scoring was done by a judge who was blind to the purposes of the experiment. The judge was trained in the scoring procedure until a satisfactory level of agreement was achieved between the judge and author (kappa $>.8$ ).

\section{RESULTS}

Two sets of analyses were performed. The level of significance exceeds .05 in all tests unless indicated otherwise. The first set of analyses compared recall for the signal and no signal conditions. More idea units were recalled for the text with signals $(49.16 \%$ of the ideas) than for the text without signals $(44.88 \%)[t(111)=2.00]$. Recall of information associated with number signals was higher for the text with signals (56.55\%) than for the text without signals $(45.81 \%)$. This effect was reliable when tested over subjects $[t(111)=3.13]$ and when tested over items $[\mathrm{t}(8)=2.53]$. Nonspecific recall was not reliably different for the signal $(47.22 \%)$ and no signal conditions $(44.63 \%)[\mathrm{t}(111)=1.09, \mathrm{p}>.1]$.

The second analysis concerned individual differences in signaling effects. In this analysis, the specific recall scores were interpreted as a measure of the signaling effect because only those scores were affected by the signaling manipulation. The nonspecific recall scores were interpreted as a measure of subjects' text-recall abilities because there was no effect of the signaling manipulation on those scores. For each experimental condition, subjects' specific recall scores were regressed on their nonspecific recall scores. The slope coefficient was greater in the signal condition (1.157) than in the no signal condition $(0.615)[t(109)=2.51]$. Looking at it another way, when subjects within each condition were divided in half according to their nonspecific recall scores, the difference in specific recall scores between the signal and no signal conditions was greater for the good recallers ( $15.5 \%$ difference) than for the poor recallers $(3.9 \%$ difference).

\section{DISCUSSION}

The results of the experiment are easily summarized. First, the presence of organizational signals selectively aided recall of those ideas which were specifically marked by numbering signals. Further, better recallers benefitted more than poorer recallers from the presence of text signals. Let us consider the implications of these findings.

This is the first demonstation of an effect of text-embedded organizational signals on overall recall when results are averaged across all subjects. However, the effect on overall recall was primarily due to the fact that signaling effects were greater for ideas that were specifically associated with text signals than for ideas that were not specifically marked. This result of a selective effect of signaling on recall is consistent with several previous findings (Fowler \& Barker, 1974; Glynn \& DiVesta, 1979; Loman \& Mayer, 1983; Marshall \& Glock, 1978). Together, these results suggest that previous investigators failed to observe effects of organizational signals because they examined only overall recall (Britton et al., 1982; Meyer, 1975). Specific recall effects of the organizational signals may have been obscured by this procedure.

Given that signals facilitate recall of the content they mark, we might ask how the signals operate. Although the data do not resolve this issue, several alternative mechanisms may contribute to the signaling effect. First, signals may cause readers to pay more attention to the as- 
sociated content with the consequence of better memory for the information. This hypothesis might be tested by measuring reading times for signaled and unsignaled sentences or by use of a secondary probe reaction time task. Second, explicitly marking the organization of text content may lead readers to represent that organization more completely. A representation of the organization of specific text content should allow for more systematic and complete recall of the specific information. Third, signals may influence the order in which text information is recalled. Signaled information may be produced earlier in recall than unsignaled information with the consequence that signaled content is subjected to less output interference than unsignaled content. Finally, signals may distinguish the associated content from unsignaled content and confer greater importance on the signaled information. To the extent that the process of free recall entails some degree of editing of redundant and unimportant information, the effect of signals may be to make the associated information less likely to be deleted from recall.

The second finding of the experiment was that the effect of the organizational signals depended upon the text-recall ability of the subject. One implication of this result is that previous investigations may have failed to find effects of organizational signals in part because they averaged results across subjects of differing abilities. The more important implications of the finding concern the cognitive basis for the relationship.

Two competing hypotheses were offered concerning the relation between text-recall ability and the effects of signaling. First, if good and poor recallers differ in how actively they employ organizational strategies during reading, then including explicit signals to text organization should have benefitted the passive, poorer recallers more than the good recallers. Alternatively, good recallers may construct more coherent text representations because they employ available information to better organize their memories. The results supported the second hypothesis: Subjects who were better recallers benefitted more from the organizational signals than poorer recallers. This result is similar to a previous finding that underlining affected the recalls of slow learners less than the recalls of medium or fast learners (Crouse \& Idstein, 1972).

Finally, the preliminary nature of these findings and conclusions should be noted. First, the findings are based upon one text. They must be replicated with different texts before they can be generalized. Second, it is tempting to attribute the observed selective recall effects specifically to the number signals rather than to the combined effects of the number signals and preview sentences. However, the design of the experiment does not allow such a conclusion. Future research should manipulate the two types of signals independently. Third, the relation between signaling effects and recall ability must be tested using a measure of recall ability which is defined a priori and independently of the experimental manipulations. Finally, other types of organizational signals must be systematically investigated. This is a particularly important issue given the finding that some organizational signals appear to benefit underachieving readers more than good readers (Meyer et.al., 1980), in apparent contrast to the findings of the current study.

\section{REFERENCES}

Britton, B. K., Glynn, S. M., Meyer, B. J. F., \& Penland, M. J. (1982). Effects of text structure on use of cognitive capacity during reading. Journal of Educational Psychology, 74, 51-61.

Brooks, L. W., Dansereau, D. F., Spurlin, J. E., \& Holley, C. D. (1983). Effects of headings on text processing. Journal of Educational Psychology, 75, 292-302.

Brown, A. L. (1980). Metacognitive development and reading. In R. J. Spiro, B. C. Bruce, \& W. F. Brewer (Eds.), Theoretical issues in reading comprehension (pp. 453-481). Hillsdale, NJ: Erlbaum.

Brown, J. I., Nelson, M. J., \& Denny, E. C. (1973). The NelsonDenny reading test. Boston: Houghton Mifflin.

Crouse, J. H., \& IDstein, P. (1972). Effects of encoding cues on prose learning. Journal of Educational Psychology, 63, 309-313.

FOWLER, R. L., \& BARKER, A. S. (1974). Effectiveness of highlighting for retention of text material. Journal of Applied Psychology, 59, 358-364.

Frase, L. T. (1969). Paragraph organization of written materials: The influence of conceptual clustering upon the level and organization of recall. Journal of Educational Psychology, 68, 394-401.

GlynN, S. M., \& DiVesta, F. J. (1979). Control of prose processing via instructional and typographical cues. Journal of Educational Psychology, 71, 595-603.

IRWIN, J. W. (1982). The effects of coherence explicitness on college readers' prose comprehension. Journal of Reading Behavior, 14, 275-284.

KINTSCH, W., \& VAN DiJK, T. A. (1978). Toward a model of discourse comprehension and production. Psychological Review, 85, 363-394.

LOMAN, N. L., \& MAYER, R. E. (1983). Signaling techniques that increase the understandability of expository prose. Journal of Educational Psychology, 75, 402-412.

Marshall, N., \& Glock, M. D. (1978). Comprehension of connected discourse: A study into the relationship between the structure of text and information recalled. Reading Research Quarterly, 14, 10-56.

MEYER, B. J. F. (1975). The organization of prose and its effects in memory. Amsterdam: North-Holland.

MeYer, B. J. F., BRANDT, D. M., \& BLUTH, G. J. (1980). Use of toplevel structure in text: Key for reading comprehension of ninth-grade students. Reading Research Quarterly, 16, 72-102.

Tulving, E., \& PEARlstone, Z. (1966). Availability versus accessibility of information in memory for words. Journal of Verbal Learning \& Verbal Behavior, 5, 281-291.

VAN DiJK, T. A. (1979). Relevance assignment in discuurse comprehension. Discourse Processes, 2, 113-126.

Wallechinsky, D., \& WAllace, I. (1978). The people's almanac \#2. New York: Bantam Books.

(Manuscript received for publication April 11, 1985.) 